Tributación y Objetivos de Desarrollo Sostenible. Policy-making en Tributación, Cooperación tributaria Internacional y Gobernanza Fiscal Mundial como principal fuente de financiación de la Agenda 2030 de las Naciones Unidas. Proyecto (Dirección: Jeffrey OWENS, Michael LENNARD, Eva ANDRÉS)

\title{
[II PARTE]
}

"Taxation and Sustainable Development Goals. Policy-making on Taxation, International Tax Cooperation and Global Tax Governance as a main financial source of 2030 UN. Project (Dirs. Jeffrey OWENS, Michael LENNARD, Eva ANDRÉS)

\section{[PART II]}

\section{Jeffrey Owens}

Professor of International Tax Law

Director of the Global Tax Policy Center of Vienna

Institute for Austrian and International Tax Law

University of Vienna

E-mail: jeffrey.owens@wu.ac.at

\section{Michael Lennard}

Chief of International Tax Cooperation in the Financing for Development Office of the UN

Secretary, Committee of experts on International

Cooperation in Tax Matters

E-mail: lennard@un.org

Eva Andrés Aucejo

Catedrática de Derecho Financiero y Tributario

Directora de la Revista de Educación y Derecho.

Departamento de Derecho Administrativo, Derecho Procesal y

Derecho Financiero y Tributario

Universidad de Barcelona, España

E-mail: eandres@ub.edu 
TAXATION AND SUSTAINABLE DEVELOPMENT GOALS (TAXATION\&SDG): "Rule \&Policy-making on <Taxation>, <International Tax Cooperation> and <Global Tax

Governance > as a main financial source of the SDG 2030 UN \& A. ABABA Agendas", Project.

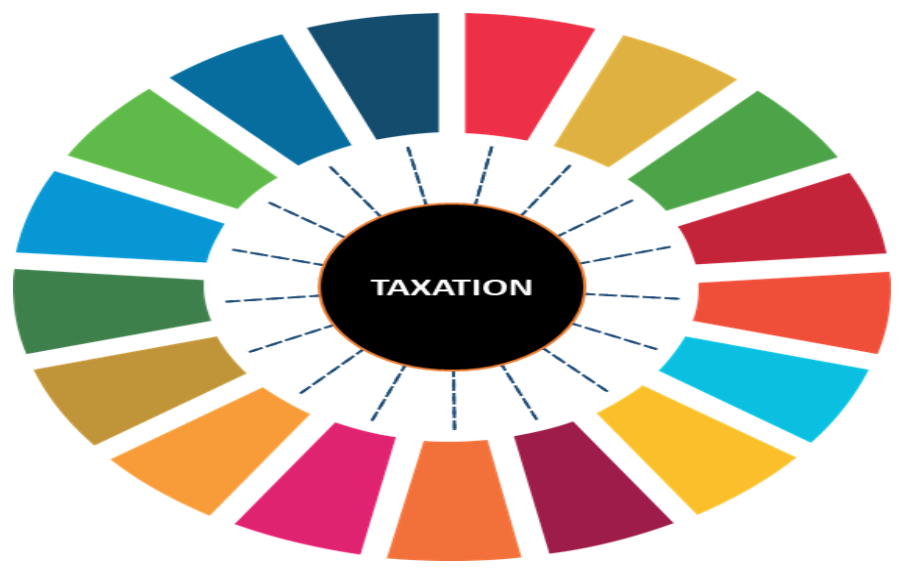

\section{DIRECTORS:}

Jeffrey Owens

Director of WU Global Tax Policy Center. Vienna

Michael Lennard

Secretary of UN Tax Committee. New York

Eva Andrés

Professor of Tax Law. UB

PRINCIPAL INVESTIGATOR:

Eva Andrés

WB/OECD HCBM Project, Consulter

SCIENTIFIC COMMITTEE: J. Owens, M. Lennard, E. Andrés, M. Nicoli, J. Sen, S. Bokobo,M. Callijuri, C. García-Herrera, S. Díaz de Serralde, P. Valente ADVISORY COMMITTEE: J. Martín Queralt, S. Raventós, William Byrnes, George Salis, G. Orón, JP. Pont, A. Olesti, X. Fernández Pons 
With the cooperation of some representative members from the following International Organizations, Universities and Companies:

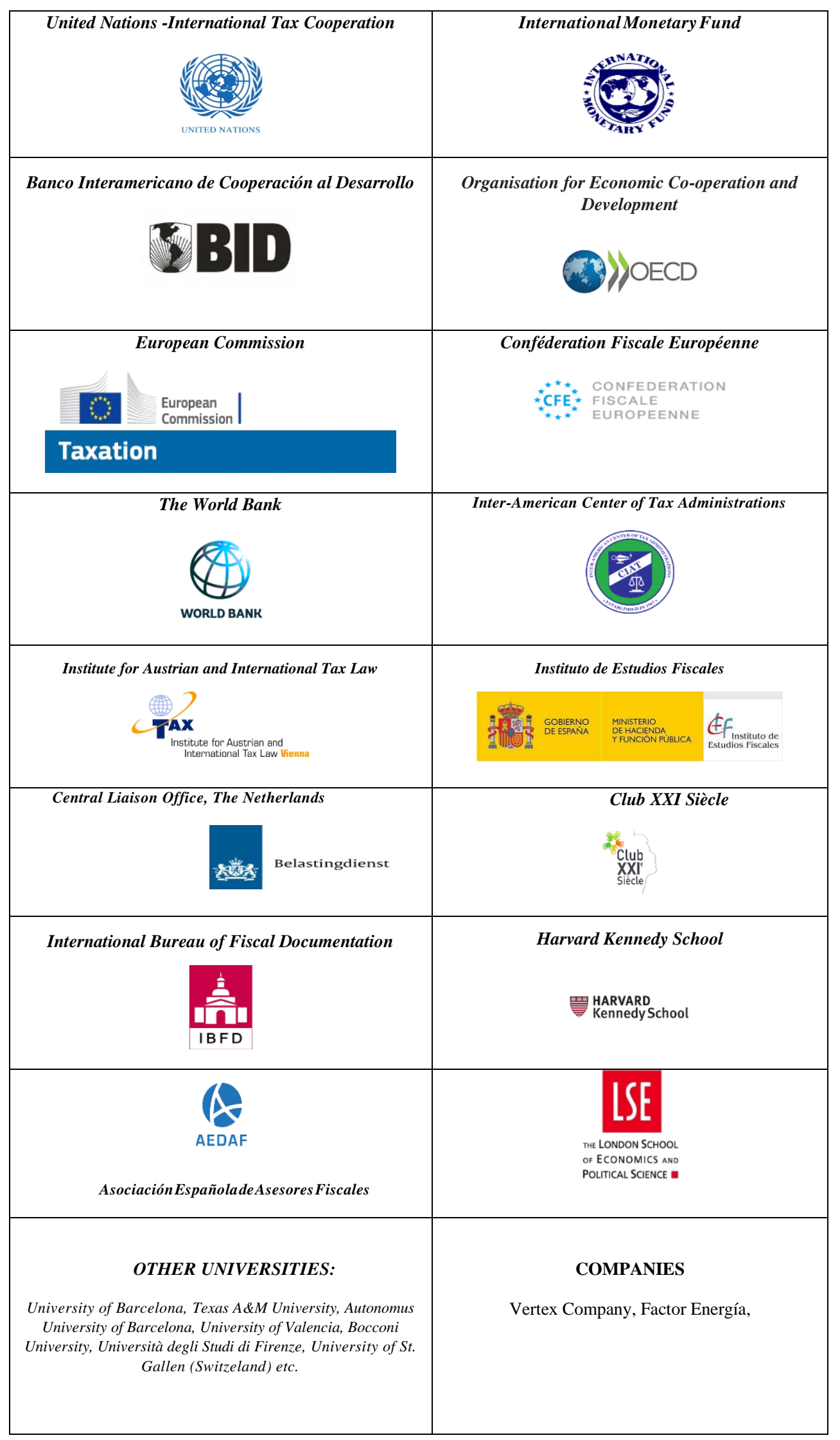




\section{BACKGROUND:}

El proyecto referido titulado Taxation and Sustainable Development Goals. Policy-making on Taxation, International Tax Cooperation and Global Tax Governance as a main financial source of $2030 \mathrm{UN}$, tiene como antecedente un histórico de investigaciones científicas derivadas de proyectos de composición internacional y redes de investigación previas en materia de cooperación fiscal internacional y gobernanza fiscal mundial, congresos y conferencias nacionales e internacionales.

Nos gustaría expresar nuestro máximo agradecimiento a las entidades que soportan este proyecto, así como a sus miembros representantes que forman parte del Comité Científico del mismo. A saber: The Global Tax Policy Center of Vienna (J. Owens); UN International Tax Committee (M. Lennard), Instituto de Estudios Fiscales, Ministerio de Hacienda de España (Cristina García-Herrera Blanco); Centro Interamericano de Administraciones Tributarias (Santiago Díaz de Serralde) y Asociación Club Siglo XXI para la diversidad (Iolanda Piedra) y, en general, al resto de miembros de comités científicos y comités asesores, así como a todos los investigadores que forman parte del mismo.

En relación a sus antecedentes, nuestros proyectos previos se gestaron desde sus orígenes con reconocidas Organizaciones Internacionales, Centros de investigación Internacionales, Ministerios de Hacienda (Instituto de Estudios Fiscales de España y Agencia Tributaria de Netherlands) / Departament Hisenda, Generalitat de Catalunya y Universidades nacionales e internacionales, con expertos reconocidos en la materia, lo que ha generado relevantes inputs y outputs.

Por cuanto respecta a nuestra colaboración con Organizaciones Internacionales, la Red de Excelencia que actualmente lideramos desde la Universidad de Barcelona: "The global observatory on Tax Agencies: towards International Tax Cooperation and Global Tax Governance es partner del "Global forum on Law, Justice and Development, del Banco Mundial, a través del proyecto Human-Centered Business Model creado por el Banco Mundial y actualmente también bajo el paraguas de la OCDE. ${ }^{1}$

La investigadora principal, Dra. Andrés es consultora del citado proyecto del Banco Mundial, proyecto que, actualmente está siendo desarrollado por la OCDE, en cuya sede de París hemos tenido ocasión de participar en reuniones al efecto.

\footnotetext{
${ }^{1}$ http://www.ub.edu/web/ub/en/menu_eines/noticies/2017/06/031.html
} 

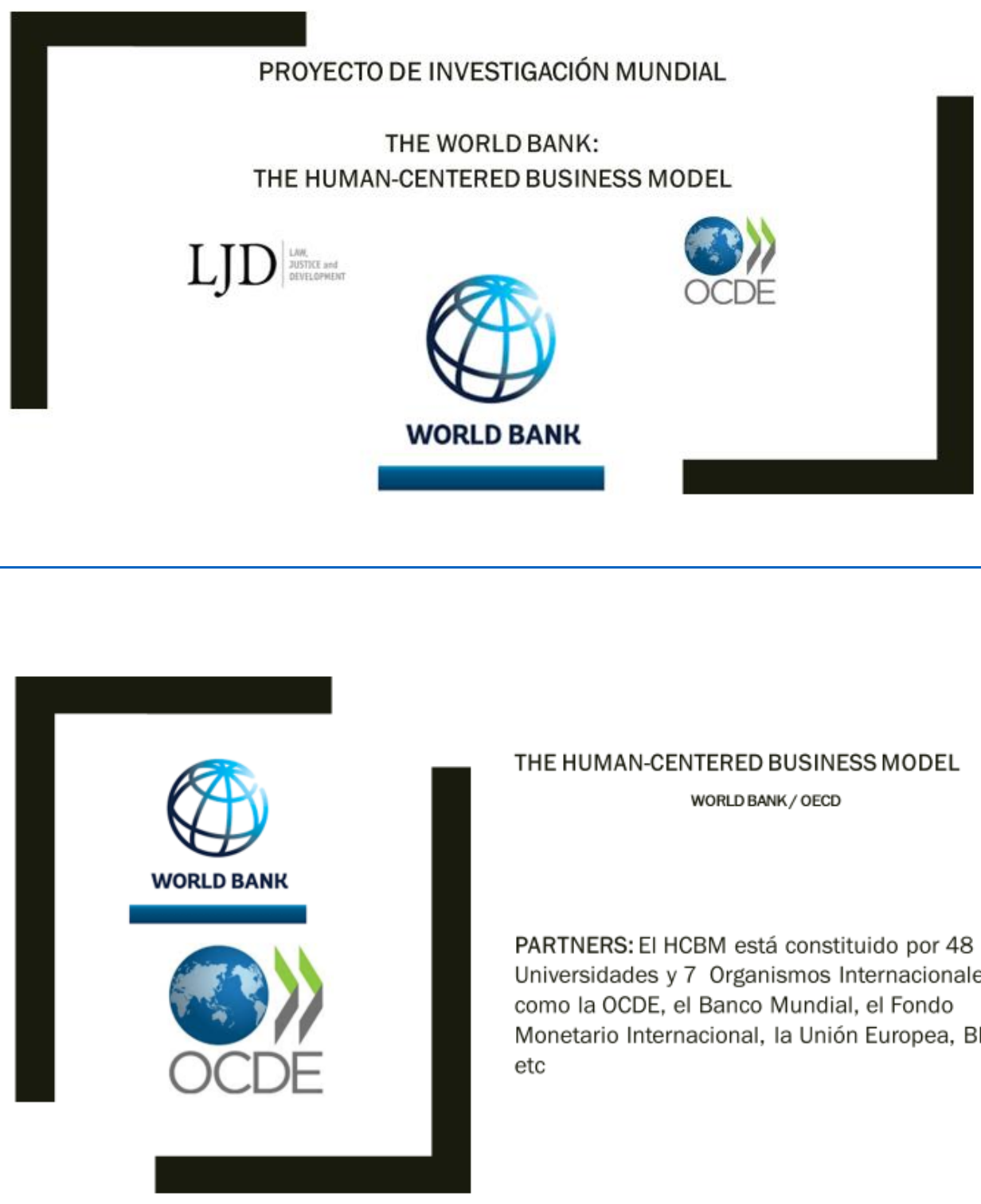

THE HUMAN-CENTERED BUSINESS MODEL

WORLDBANK/OECD

PARTNERS: EI HCBM está constituido por 48 Universidades y 7 Organismos Internacionales como la OCDE, el Banco Mundial, el Fondo Monetario Internacional, la Unión Europea, BID, etc

Desde aquí nos gustaría destacar las invitaciones y la participación de miembros del Fondo Monetario Internacional que están liderando el programa Tax Administration Diagnostic Assesment Tool (TADAT) en nuestras conferencias internacionales y otros feedbacks que hemos mantenido con los directores del programa TADAT del Fondo Monetario Internacional, en su sede física de Washington, con ocasión de nuestra estancia de investigación realizada en la Universidad de Georgetown y en el propio Banco Mundial (Vicepresidencia), ambos también con sede Washington.

E igualmente significamos muy positivamente nuestra participación en las Sesiones del Comité de Expertos de Cooperación Fiscal Internacional de la ONU, UN Tax Committee, sesiones que se realizan semestralmente en las sedes de la ONU de Ginebra y Nueva York, así como en sesiones 
del Consejo Económico y Social de las Naciones Unidas (ECOSOC), en New York, quienes también están desarrollando un extraordinario trabajo.

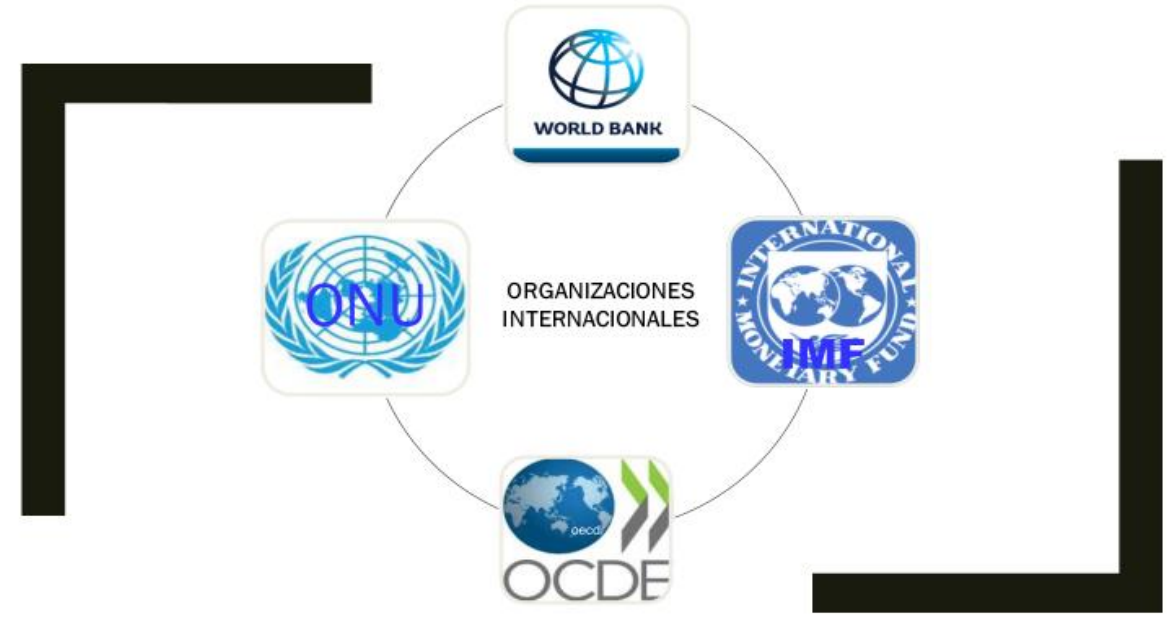

Nos gustaría también poner en valor las alianzas establecidas con algunos de los Centros de Investigación de Fiscalidad internacional más importantes del mundo, como son el Global Tax Policy Center de Viena, o también el International Bureau of Fiscal Documentation, así como la reconocida Confederación Fiscal Europea, con sede en Bruselas, instituciones partners de la Universidad de Barcelona en proyectos que hemos liderado. También hemos colaborado con Asociaciones internacionales y europeas de gran envergadura, especializadas en fiscalidad internacional desde Europa y desde América.

La autora de esta presentación es también, entre otros, investigadora del Proyecto del World Tax Center of Vienna: Multi-Stakeholder Group on Improving Cross-border Dispute Resolution. Dirección: Jeffrey Owens.

\begin{tabular}{|l|l|}
\hline $\begin{array}{l}\text { WU GLOBAL TAX POLICY CENTER (Vienna), dirigido por } \\
\text { JEFFREY OWENS, director de gran parte de los proyectos y también } \\
\text { institución partner de nuestros proyectos }\end{array}$ \\
\hline $\begin{array}{l}\text { INTERNATIONAL BUREAU OF FISCAL DOCUMENTATION. } \\
\text { EL IBFD, Institutición Partner, siendo muchos de sus investigadores } \\
\text { colaboradores habituales de nuestros proyectos }\end{array}$ \\
\hline $\begin{array}{l}\text { LA CONFEDERACIÓN FISCAL EUROPEA } \\
\text { CONFÉDÉRATION FISCAL EUROPÉENNE (CFE) }\end{array}$ \\
\hline $\begin{array}{l}\text { INTER-AMERICAN CENTER FOR TAX } \\
\text { ADMINISTRATION }\end{array}$ \\
\hline
\end{tabular}




\section{INTRA-EUROPEAN ORGANIZATION OF TAX} ADMINISTRATIONS (IOTA)

Son asimismo reseñables las importantes sinergias que hemos tenido con Universidades internacionales y nacionales con las cuales hemos podido trabajar conjuntamente y avanzar en muchos de los problemas y retos que tiene actualmente la fiscalidad internacional. Así destacamos, a título de ejemplo: la Universidad de Oxford, London School of Economics, la Kennedy School of Government de Harvard, Universidad de Zurich, University of Vienna, University of Texas, Università di Bologna, Università degli Studi di Firenze, Università Link de Roma, University of Leeds, University of Durham, etc.

Además, hemos mantenido constantes sinergias y colaboraciones con la Agencia Tributaria Española, el Instituto de Estudios Fiscales, la Asociación Española de Asesores Fiscales, la Asociación Española de Derecho Financiero. Además en el ámbito de Comunidad Autónoma hemos participado con el Departament de Hisenda de la Generalitat de Catalunya, el Departament de Affers Socials, Pymec, entre otros, así como con un importante número de investigadores de Departamentos de Derecho Financiero y Tributario y Derecho Internacional Público de España.

A todos los entes y personas que han venido participando y participan en nuestros proyectos nos gustaría mostrar nuestro más sincero agradecimiento y nuestra invitación sincera para seguir haciéndolo en el futuro. Sin duda es un honor, una satisfacción y un placer mantener esta línea de cooperación y colaboración recíproca.

Entre los proyectos a destacar, que han dado pie al presente proyecto internacional son de destacar los siguientes proyectos competitivos:

DER.15-68768-P Project: International Administrative Co-Operation in Tax Matters and ADR of Transnational Tax Disputes and Models for an Institutional Architecture from a European Perspective- EUDISCOOP PROJECT (2015-2019) (I.P. Eva Andrés) ${ }^{2}$

\footnotetext{
2 Proyecto valorado por el Comité de expertos del Ministerio con la máxima calificación, habiéndose destacado su excelencia en diseño, publicaciones e internacionalización.

International Congress (2017): International Administrative Co-operation in Tax Matters and Tax Governance, http://www.ub.edu/dret-butlleti/29-11-16/International_Congress_2017_desglosado.pdf; http://diposit.ub.edu/dspace/handle/2445/103284

Report of the International Congress 2017: "International Administrative Cooperation in Tax Matters and Tax Governance". On Barcelona, January 26th, 2017. Facultad de Derecho. Universidad de Barcelona; Chronicle of International Congress 2017: "International Administrative Cooperation in Tax Matters and Tax Governance". On Barcelona, January 26th, 2017. Facultad de Derecho. Universidad de Barcelona, Book of the Conference Proceedings: International Congress 2017.International Administrative Cooperation in Tax Matters and Tax Governance

(2018) INTERNATIONAL CONGRESS: GLOBAL TAX ADMINISTRATIONS' EFFICIENCY: INTERNATIONAL FISCAL COOPERATION AND GOVERNANCE, (2018) Institute of Fiscal Studies-
} 

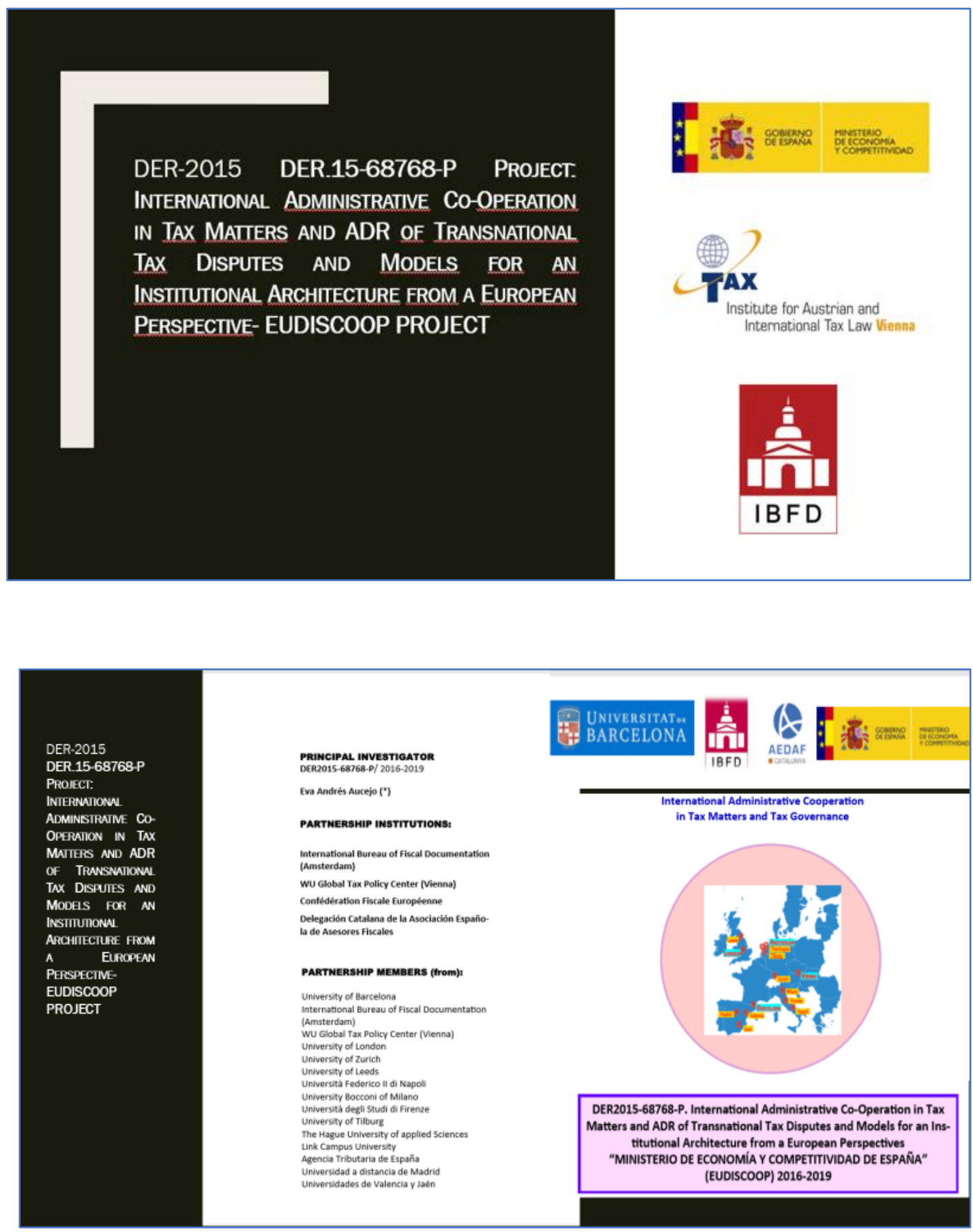

Madrid,

https://derecho.ucm.es/data/cont/docs/23-2018-05-28Programa\%20IEF\%2030\%20mayo\%202018\%20vdef2505.pdf.

La eficacia de las Administraciones tributarias en el marco de la gobernanza fiscal internacional; UNIVERSIDAD COMPLUTENSE DE MADRID.

https://derechofinancieroytributario.files.wordpress.com/2018/05/programaxtax-ucm-31-mayo-2018vdef2205.pdf.

International Congress. Administración Publica de Cataluña, 1 de junio de 2018, https://www.ub.edu/portal/web/dret/detall/-/detall/01-06-de-8-45-a-15-30-h-congres-internacional-globaltax-administration-efficiency-international-fiscal-cooperation-and-governance-a-l-escola-d-admini;

«International Tax Cooperation» Congress 2019: Digital Economy, Transfer Pricing and Litigation in Tax Matters (MAPs + ADR). Ongoing 2030 (SDG) and Addis Ababa Agendas; https://www.ub.edu/portal/documents/620105/0/L10-+INTERNATIONAL+TAX+CONGRESS-2019BCN.pdf/885cec55-f863-b119-d759-34ef6a1e94f8 


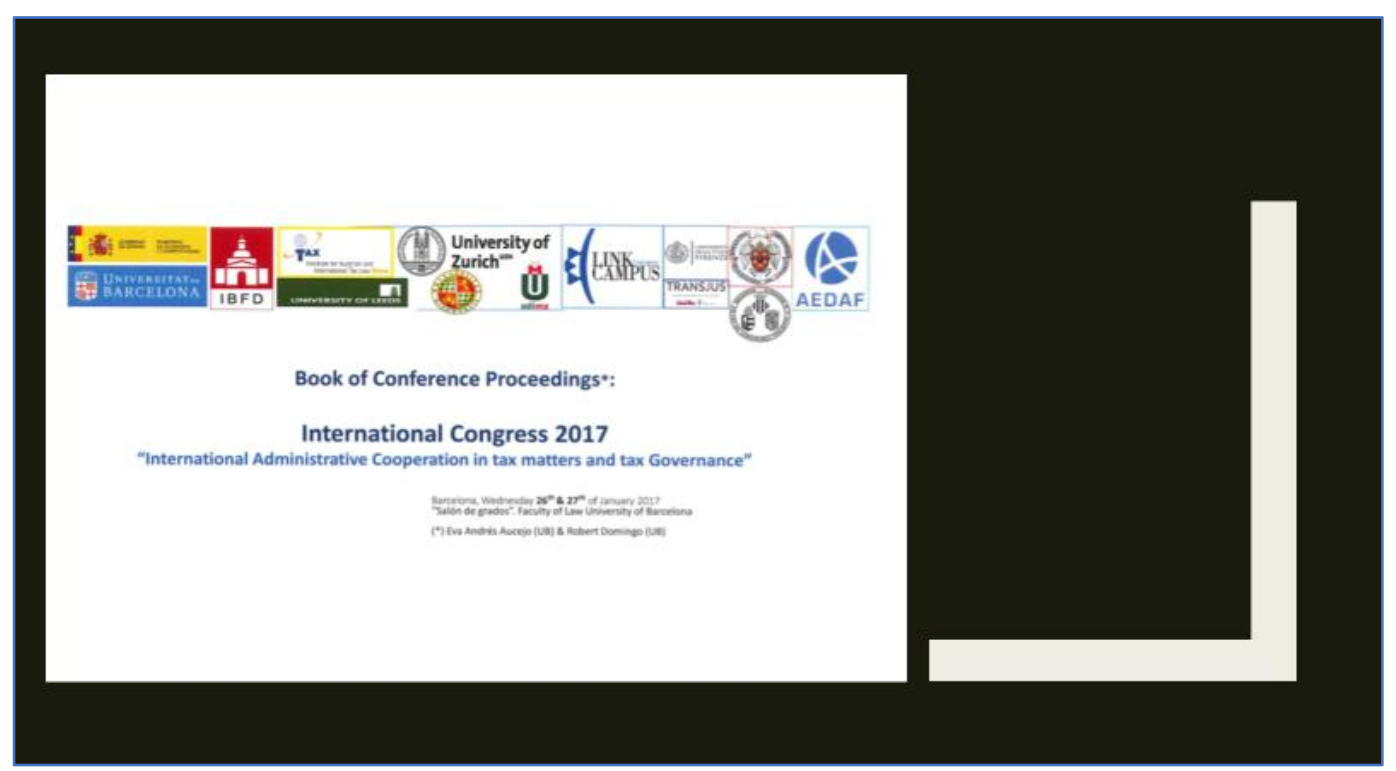

Book of the Conference Proceedings: International Congress 2017. International Administrative Cooperation in Tax Matters and Tax Governance http://diposit.ub.edu/dspace/handle/2445/103284

The Excellence Network DER 2017-90874-REDT -GOTA-INTAXCOOP \& GOV: The Global Observatory on Tax Agencies: Towards on the International Tax Cooperation and Global Governance (P.I.: Eva Andrés)

DER 201790874-REDT GOTAINTAXCOOP\& GOVERNANCE

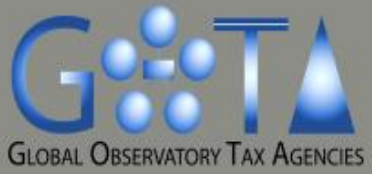

\section{EXCELLENCE NETWORK}

THE GLOBAL OBSERVATORY ON TAX AgENCIES: TOWARDS TO THE INTERNATIONAL TAX

COOPERATION AND GLOBAL TAX GOVERNANCE

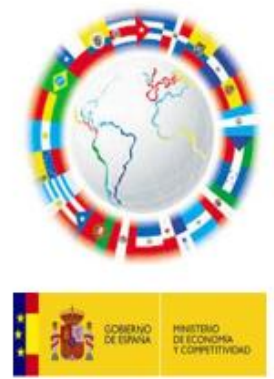




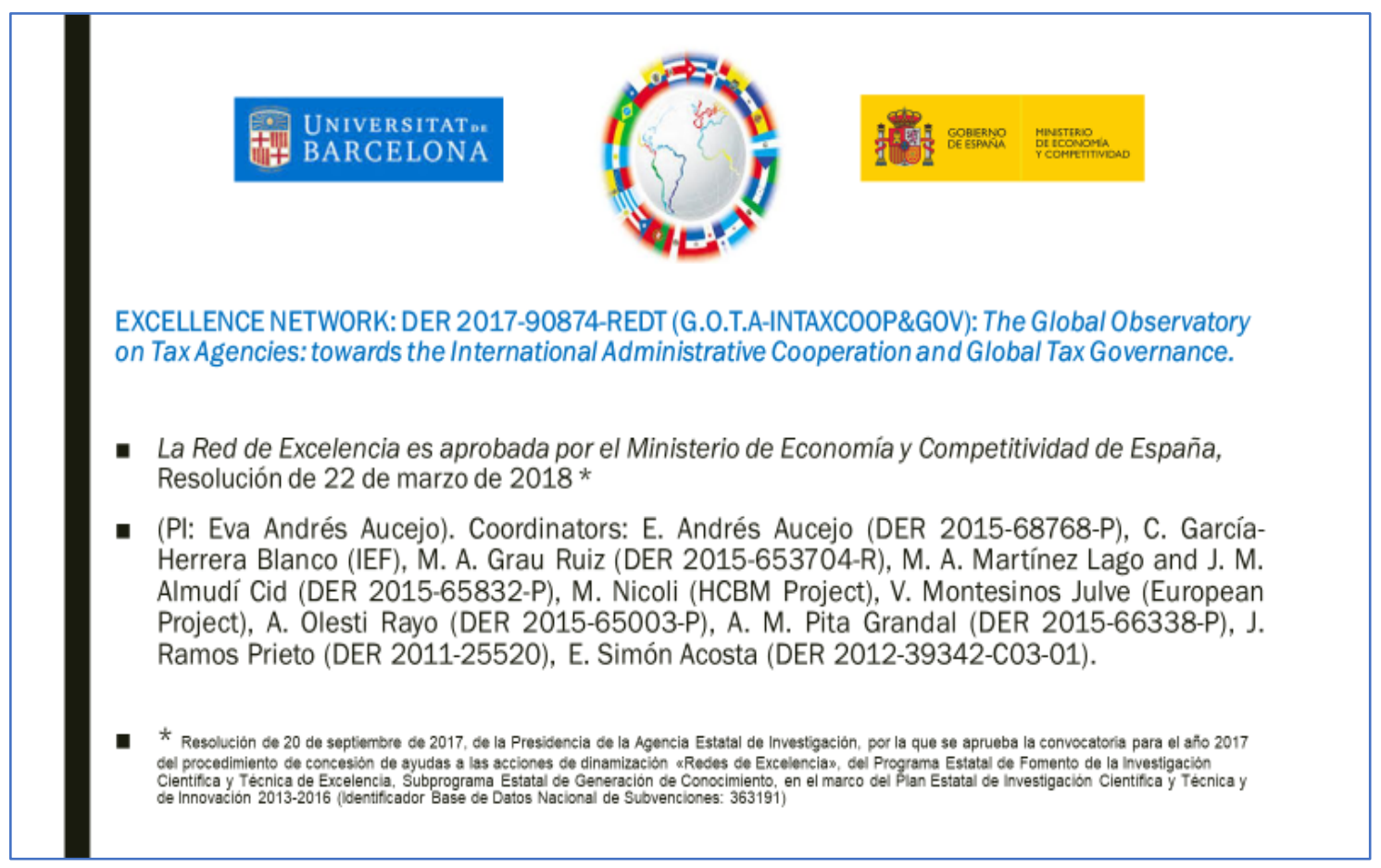

Mediante la Red de Excelencia (THE GLOBAL OBSERVTORY ON TAX AGENCIES (G.O.T.A- DER 2017- 90874-REDT INTAXCOOP\&GOV): proyectada sobre los tres ejes que siguen: - La Eficiencia de las Administraciones Tributarias Mundiales; - La Cooperación Internacional de las Administraciones Tributarias y - la Gobernanza Fiscal Mundial), hemos realizado colaboraciones recíprocas con Organismos Internacionales Gubernamentales y no Gubernamentales, abordando análisis y evaluación de Modelos de Eficiencia de las Administraciones tributarias mundiales, como por ejemplo: - EI FONDO MONETARIO INTERNACIONAL: TADAT (financiado por la UE, la Agencia tributaria de Netherlans, Japón, UK...), sobre diagnóstico y evaluación de las Agencias tributarias; - La Agenda de la INTRAEUROPEAN ORGANIZATION OF TAX ADMINISTRATIONS (IOTA) u Organización Intra Europea de Administraciones tributarias de Europa; - La INTER-AMERICAN CENTER FOR TAX ADMINISTRATION - CIAT (para Latino América), La CONFEDERACIÓN FISCAL EUROPEA, etc, con una participación muy significada de la Agencia tributaria de Países Bajos, la Agencia Tributaria Española y el Instituto de Estudios Fiscales, el Departamento de Hacienda de la Generalitat de Catalunya, las Universidades de Oxford, Kennedy School of Government de Harvard, la London School of Economics and Political Sciences, la Università Link de Roma, Università degli Studi di Firenze, Universidad Autónoma de Chile, entre otras.

La autora de esta presentación, Dra. Andrés ha sido también propuesta para la Dirección de una Cátedra mundial sobre Cooperación Fiscal Internacional y Gobernanza Mundial denominada: 
"UB - C. XXI Siècle" GLOBAL Chair on International Tax Cooperation and Global Tax Governance: GLOBAL TAX POLICY MAKING \& SDG (UN 2030 Agenda, AAAA, Doha, Monterrey Consensus) and Education \& SDG (UN 2030 SDG. N-4)

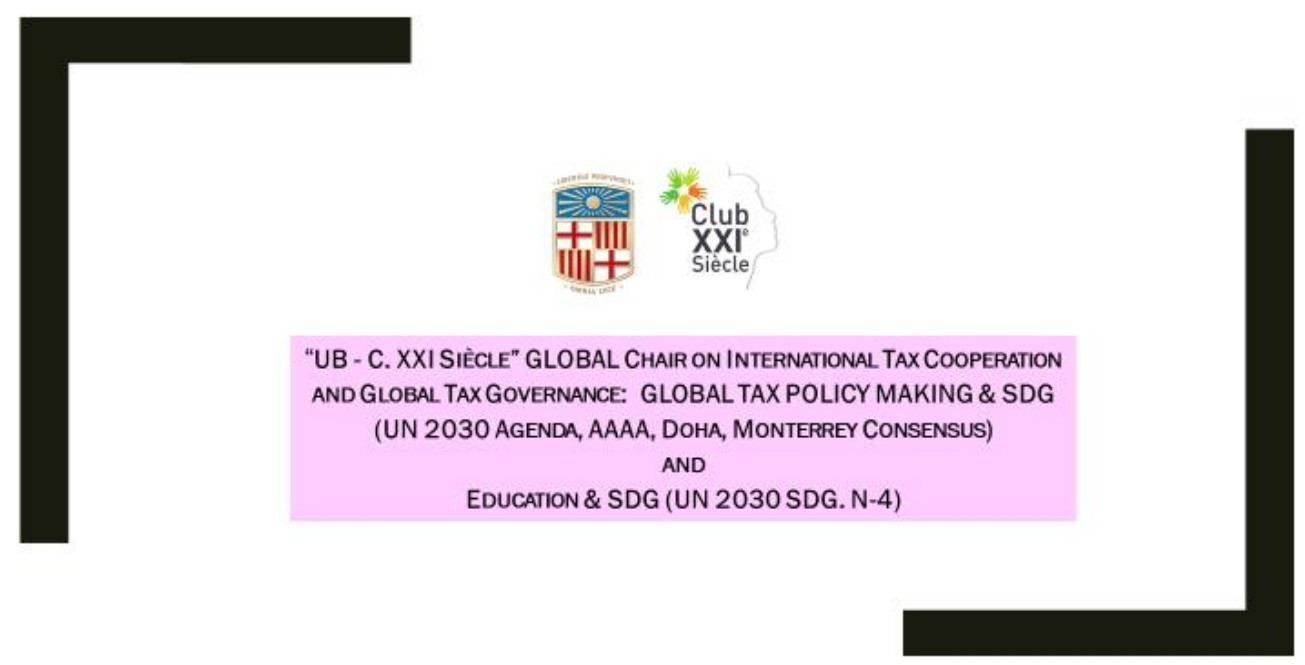

"UB - C. XXI SIĖCLE" CHAIR ON INTERNATIONAL TAX COOPERATIONAND GLOBAL TAX GOVERNANCE: POLICY MAKING ON TAXATION \& SDG (UN 2030 AND A. ABABA AgENDAS) AND EDUCATION \& SDG (UN 2030 SDG. N-4)
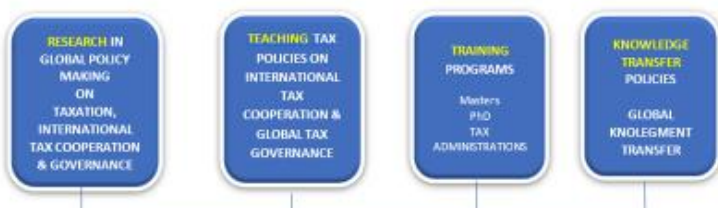

UB-C. XOO SUECLE CHAIR ON INTERNA TONAL TAX COOPERATION AND GLOBAL TAX GOVER. NANCE. ONOONG 2030 UN 8 ABABA AGENDAS: Research in policy making on TAXATON \& SDO 
La Cátedra “UB -C.XX Siècle Chair on International Tax Cooperation and Global Tax Governance. Policy making on Taxation \& SDG (UN 2030 and A. Ababa Agendas). Education \& SDG (UN 2030 SDG. N-4)", tiene por objeto constituir una plataforma para la investigación docencia y transferencia sobre creación de políticas fiscales públicas y público-privadas (transversales, inclusivas y sostenibles) en los grandes retos que tiene hoy pendiente de acometer la Fiscalidad Internacional/mundial, destinadas a lograr la Cooperación Internacional entre todos los países, así como a diseñar una arquitectura de Gobernanza fiscal mundial, en línea con las grandes metas mundiales recogidas en las Agendas 2030 de Naciones Unidas (ODS), la Agenda Addis Abeba, la Conferencia de Monterey y Doha Declaration.

Amén del gran objetivo general anterior, esta cátedra tendrá un segundo gran objetivo bajo el título: Educación y Objetivos de Desarrollo Sostenible (UN 2030 SDG. N-4), consistente en la creación de políticas científicas en investigación legal, docencia y transferencia hacia la consecución de las metas del objetivo número 4 de los Objetivos de Desarrollo sostenible de la Agenda 2030 de Naciones Unidas, que lleva por rubro "Educación de calidad", incidiendo en el derecho a la Educación Superior en todas sus aristas. A saber: en la Educación como bien fundamental ínsito en las líneas directrices de las políticas públicas y público-privadas; en la Educación como Derecho Humano reconocido mundialmente en la Declaración de Derechos Humanos, en la Educación como Derecho fundamental reconocido en las constituciones comparadas de los Estados democráticos; en la "Educación en Valores" como forma de alcanzar otras metas de los Objetivos de Desarrollo Sostenible, en la Internacionalización de la Educación Superior y también en Educación y Fiscalidad, ahondando en las políticas públicas que deben presidir una buena formación en materia de fiscalidad y hacienda pública hacia la consecución de los bienes y servicios de necesidad colectiva. También desde este gran segundo objetivo general se enfocará su acometido tanto desde la investigación científica, como desde el diseño de políticas educativas para su posible implementación en los programas de grado y/o tercer ciclo, políticas formativas y de transferencia de los conocimientos científicos citados.

Una tercera variante a la que sin duda, investigadoras e investigadores de la Cátedra institucional dedicarán su atención es el Género desde sus distintas aristas. La excelente presentación que se hace en este número 21 de la Revista Educación y Derecho, escrito 
por las Catedráticas de la Universidad de Barcelona MAITE VILALTA (Vicerrectora de Igualdad UB) y PILAR RIVAS (catedrática de Derecho Laboral), son una buena muestra de ello.

Damos cierre a esta breve presentación señalando como output del incipiente proyecto descrito la inminente Jornada nacional que tendrá lugar en la Universidad de Barcelona, titulada Global Tax Policy Seminar 2020 «Policy-making on International Economic Law Conference», que se celebrará cuando termine el estado de alerta decretado por el Gobierno de España como consecuencia de la pandemia provocada por el COVID-19.

Para más información:

https://www.ub.edu/portal/web/dret/detall/-/detall/01-04-de-9-15-a-14-30-h-global-taxpolicy-seminar-2020-policy-making-on-international-economic-law-conference-

No sin antes dejar al menos referencia en estas últimas líneas de la extraordinaria Conferencia mundial con treinta y cinco delegaciones mundiales que tendrá lugar próximamente en Viena, bajo el liderazgo del profesor Owens, director del Global Tax Policy Center, de la Universidad de Viena sobre "Tax Transparency and Corruption" Conference organizada por el Institute for Austrian and International Law of Vienna in association with the World Bank Group and African Tax Institute. 